Case Report

\title{
Osteochondroma of the coronoid process of the mandible
}

\author{
Kunihiko Sawada ${ }^{1,2)}$, Dirk Schulze ${ }^{1,3)}$, Kunihito Matsumoto ${ }^{1,2)}$, Shigenori Hirai1), \\ Koji Hashimoto ${ }^{1,2}$, and Kazuya Honda ${ }^{1,2}$
}

\author{
1)Department of Oral and Maxillofacial Radiology, Nihon University School of Dentistry, Tokyo, Japan \\ 2)Division of Advanced Dental Treatment, Dental Research Center, Nihon University School of Dentistry, \\ Tokyo, Japan \\ 3)Digital Diagnostic Center, Freiburg, Germany
}

(Received June 1, 2015; Accepted September 19, 2015)

\begin{abstract}
We describe a rare case of osteochondroma of the coronoid process of mandible accompanied by severe trismus in a 14-year-old Japanese boy. The patient had initially been diagnosed as having internal derangement of the temporomandibular joint (TMJ), and conservative treatment had failed to improve the symptoms. Despite extremely limited TMJ movement, panoramic radiography revealed no abnormality, but magnetic resonance imaging suggested internal derangement of the left TMJ. Five months later, the trismus worsened and computed tomography revealed a bone-like mass confluent with the coronoid process, which was finally diagnosed as osteochondroma. The patient then underwent intraoral coronoidectomy. (J Oral Sci 57, 389-392, 2015)
\end{abstract}

Keywords: osteochondroma; coronoid process; temporomandibular joint (TMJ); trismus.

\section{Introduction}

Osteochondroma is the most common benign neoplasia of the skeleton. In 1899, Jacob first described osteochondroma of the coronoid process, forming a pseudoarthrosis joint between the coronoid process and the zygomatic arch (1). The name "Jacob's disease" has

Correspondence to Dr. Kunihiko Sawada, Department of Oral and Maxillofacial Radiology, Nihon University School of Dentistry, 1-8-13 Kanda-Surugadai, Chiyoda-ku, Tokyo 101-8310, Japan Fax:+81-3-3219-8354 E-mail: sawada.kunihiko@nihon-u.ac.jp doi.org/10.2334/josnusd.57.389 DN/JST.JSTAGE/josnusd/57.389 since been applied to this condition. Osteochondroma or Jacob's disease-hyperplasia of the mandibular coronoid process - is a benign skeletal tumor that occurs rarely in the oral and maxillofacial region. It is difficult to diagnose in the coronoid process and zygomatic arch based on panoramic radiography alone. Although panoramic radiography is a widely available screening modality, the interpretation may be difficult because of superimposition of the lesion within the area of the posterior aspect of the maxillary bone. On the basis of previous case reports, we have concluded that panoramic radiography alone is inadequate for accurate diagnosis of osteochondroma of the coronoid process of the mandible. To illustrate this, we compared the diagnostic imaging findings in previous reports with those of a case we had encountered. Here, we present details of this patient with osteochondroma of the coronoid process of the mandible accompanied by severe trismus.

\section{Case Report}

A 14-year-old Japanese boy was referred to Nihon University Dental Hospital with a complaint of trismus that had persisted for the previous 2 years. He had been diagnosed as having internal derangement of the temporomandibular joint (TMJ), and received splint therapy for several months without improvement. There was no history of facial trauma or systemic disease.

On the first visit, the patient's maximum mouth opening distance was $10 \mathrm{~mm}$, with no evidence of pain or joint sound. On palpation, the left condyle barely moved during either opening or lateral movement of the jaw. Panoramic imaging showed the left condyle to have normal morphological features (Fig. 1), while 
magnetic resonance imaging (MRI) of the TMJ revealed slight anterior disc displacement without reduction of the left TMJ (Fig. 2). The primary diagnosis was internal derangement of the left TMJ, and the patient received conservative therapy (physiotherapy, splint therapy and drug administration) for 4 months thereafter.

However, 5 months after the initial visit, the patient's mouth opening distance had further decreased by about $50 \%$ and trismus had worsened. Subsequent computed tomography $(\mathrm{CT})$ revealed a bone-like mass continuous with the tip of the left coronoid process (Fig. 3A). The surface of the lesion was continuously corticated with the cortical bone of the mandible. The lesion had developed laterally, occupying the buccal space and extending near to the posterior surface of the temporal process of the zygomatic bone and the slightly bulging zygomatic arch. There was no pseudo-joint formation between the zygoma and the lesion. Three-dimensional (3D) reconstruction of the $\mathrm{CT}$ images showed a distinct bone-like mass on the left coronoid process (Fig. 3B), while T1-weighted MR imaging indicated that the surface of this heterogeneous mass was covered with thick, low-intensity line-like cortical bone (Fig. 4A). The mass showed a high signal intensity in T2-weighted imaging (Fig. 4B).

Thus the consecutive and second diagnosis was a bone tumor of the coronoid process of the mandible. The patient underwent intra-oral coronoidectomy for resection of the osteoid mass, which was found to contain a large amount of cartilage and mature bone. Pathological analysis revealed the lesion to be an osteochondroma. One month after surgery, the patient's mouth opening distance had increased to $20 \mathrm{~mm}$, and to $30 \mathrm{~mm}$ after 6 months. Panoramic imaging showed that the osteoid mass on the coronoid process had been resected successfully (Fig. 5). Up to the time of writing, no recurrence has been observed.

\section{Discussion}

An extensive search revealed approximately 35 published cases of osteochondroma of the coronoid process of the mandible since the condition was first reported by Shackelford in 1943 (1).

An enlarged coronoid process and mushroom-like osteoid mass were observed clearly by CT imaging and 3D reconstruction, which facilitated clearer diagnosis than panoramic radiographs. The features could not be clearly identified from panoramic radiographs alone because some images of the coronoid process showed osseous duplication. Indeed, interpretation of panoramic radiographs alone may present difficulties in the diagnosis of lesions of the coronoid process and zygomatic

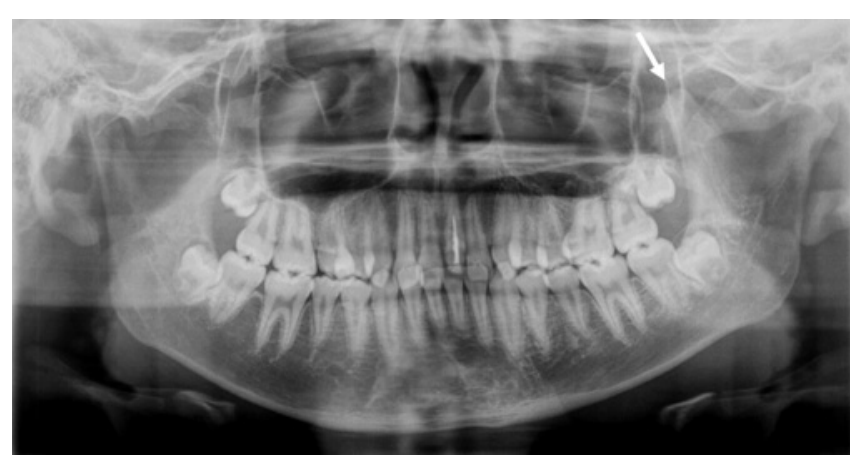

Fig. 1 Panoramic radiographic image showing normal morphology of the left coronoid process (arrow).

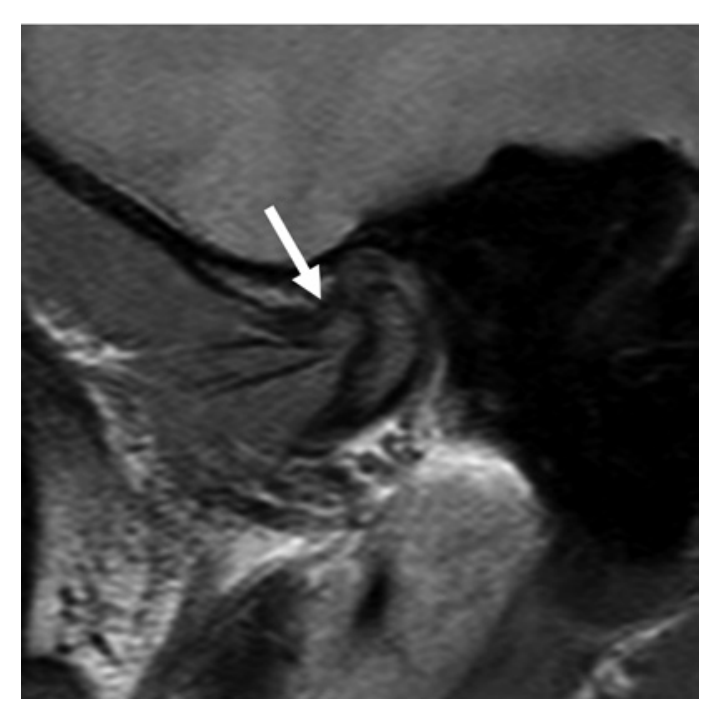

Fig. 2 Magnetic resonance imaging (MRI) revealing slight anterior disc displacement of the left temporomandibular joint (arrow). The irregular part of bone marrow signal intensity of the condyle.

arch, which can alternatively be diagnosed using CT. Totsuka et al. reported that computed tomography not only demonstrated enlargement of the coronoid process and deformity of the surrounding bones, but also disclosed the shape of the enlarged coronoid process and that of the displaced surrounding bones (2). Kerscher et al. reported that only CT demonstrated the exact shape of the enlarged coronoid process and the space between the coronoid process and surrounding bones (3). Yesildag et al. reported that $\mathrm{CT}$ imaging could be used as a guide for planning preoperative core needle biopsy and the final surgical approach (4). In addition, several authors have reported the usefulness of CT imaging (5-8). CT visualizes abnormalities of tissues and the size of the lesion and clarifies its shape and composition, location, and relationships with the neighboring structures. James et al. reported that although panoramic radiographic examination is useful for screening, superimposition of 

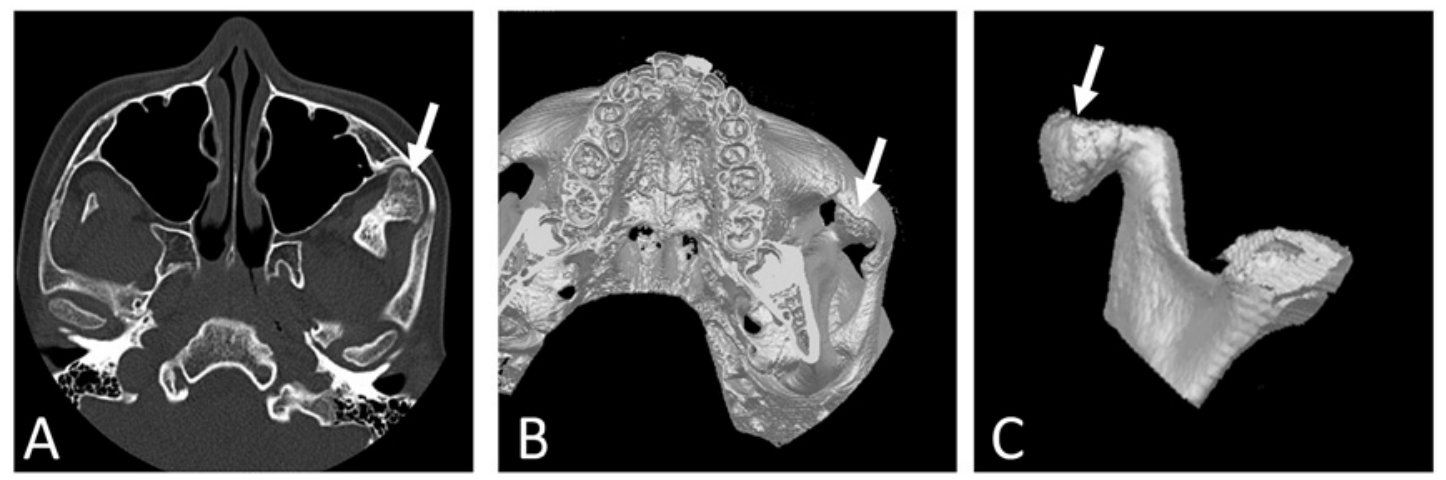

Fig. 3 (A) Computed tomography (CT) showing a bone-like mass continuous with the tip of the left coronoid process. (B) Three-dimensional CT image showing distinct bone-like mass on the left coronoid process (arrow). (C) Enlargement image of the left coronoid process (arrow).
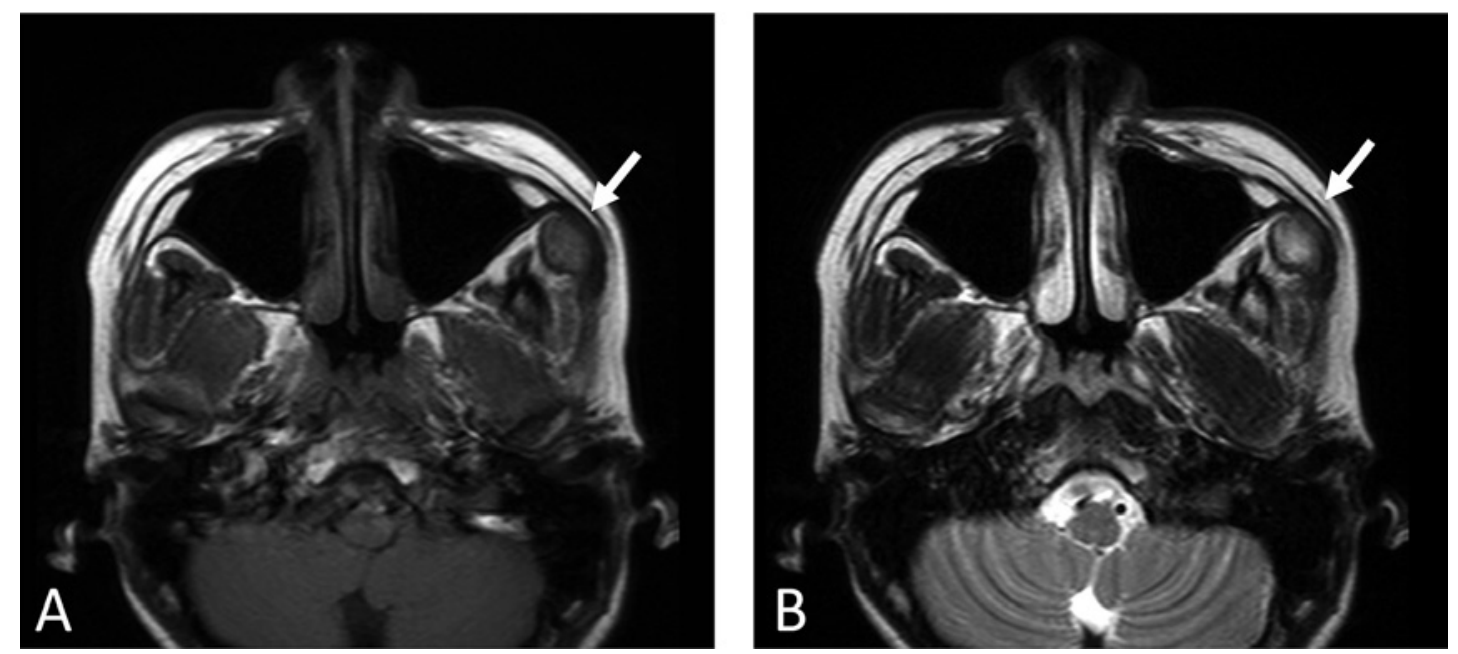

Fig. 4 (A) T1-weighted MRI image revealing thick, low-intensity line-like cortical bone over the surface of a heterogeneous mass (arrow). (B) T2-weighted image revealing a high-intensity mass (arrow).

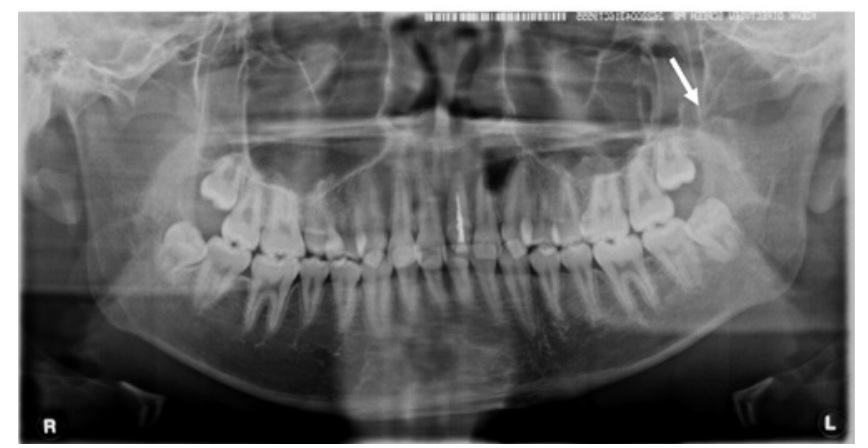

Fig. 5 Panoramic radiographic image showing successful resection of the osteoid mass from the coronoid process (arrow).

tumors can cause complications in diagnosis (5). In the present case, we ensured that the diagnosis was not based on panoramic radiography alone, and in fact CT images of the lesion were much clearer.

3D reconstruction-also often referred to as 3DCT - is essential for confirming the diagnosis, for determining the size and relationship of the tumor to surrounding structures, and for surgical planning. Emekli et al. reported that 3D reconstruction was essential for revealing the dimensions of the mass and the relationships with the surrounding structures (6). Etöz et al. reported that preoperative $\mathrm{CT}$ with $3 \mathrm{D}$ reconstruction was essential for establishing the exact extent of the lesion, and for deciding whether an intraoral or extraoral 
approach should be used (8). Tavassol et al. reported that CT with 3D reconstruction was essential for determining the correct diagnosis, and specifically for surgical planning (8). 3D reconstruction is essential for confirming the diagnosis, determining the size and relationship of the tumor to surrounding structures, and for surgical planning. We used CT imaging to confirm the presence of a mass with the same morphological characteristics as those described in previous reports. Lesions of the coronoid process can pose a diagnostic dilemma. Panoramic radiography provides adequate diagnostic information for reaching an initial diagnosis, but determination of the detailed osseous structures is dependent on X-ray incidence and exposure factors. From our experience, we believe that diagnosis is difficult using panoramic radiography alone, because imaging of the coronoid process of the mandible can reveal osseous duplication. Indeed, interpretation of panoramic radiographs may be problematic for diagnosis of lesions of the coronoid process, sectional images may be needed (i.e. CT). We recognized that other reseachers did not recommend reliance on panoramic radiography alone for diagnosis of lesions of the coronoid process.

The final pathologic diagnosis confirms hyperplasia, osteochondroma (Jacob's disease) and benign tumor of the coronoid process. Confirmation of these diagnoses is difficult based solely on a plain radiographic modality such as panoramic radiography. In our present case, CT was effective for lesion detection. However, in a patient without a history of trauma, but presenting only pain and trismus, computed tomography is not usually conducted. If the patient has severe trismus, suspected abnormality of the coronoid process based on panoramic radiography may justify the need for a CT examination. Although not used in the present case, cone-beam computed tomography (CBCT) can also be considered as a routine modality. In dentomaxillofacial radiology, $\mathrm{CBCT}$ is primarily used in place of multidetector $\mathrm{CT}$ for obtaining three-dimensional reconstructions of bone structures with a lower radiation dose. CT images can show more detailed bony growth of the coronoid process and its relationship to the zygomatic arch. CT scan, especially with 3D reconstruction, is a very useful method for diagnosis of lesions of the coronoid process. CT as well as $\mathrm{CBCT}$, and 3D reconstruction using both, are essential for completing the diagnosis, for determining the size of the tumor and its relationship to nearby structures, and for surgical planning. If the patient has severe trismus and pain with suspected abnormality of the coronoid process based on panoramic radiography, we recommend routine use of CT or CBCT for establishing a clinical diagnosis of any "suspected mass of the coronoid process".

\section{Acknowledgments}

This study was supported by the Sato Fund (2012) and a grant from the Dental Research Center, Nihon University School of Dentistry (2012), and a Grant for the Promotion of Multidisciplinary Research Projects entitled 'Translational Research Network on Orofacial Neurological Disorders' from the Japanese Ministry of Education, Culture, Sports, Science, and Technology (15K11088).

\section{References}

1. Shackelford RT, Brown WH (1943) Osteochondroma of the coronoid process of the mandible. Surg Gynecol Obstet 77, 51-54.

2. Totsuka Y, Fukuda H, Iizuka T, Shindoh M, Amemiya A (1990) Osteochondroma of the coronoid process of the mandible. Report of a case showing histological evidence of neoplasia. J Craniomaxillofac Surg 18, 27-32.

3. Kerscher A, Piette E, Tideman H, Wu PC (1993) Osteochondroma of the coronoid process of the mandible. Report of a case and review of the literature. Oral Surg Oral Med Oral Pathol 75, 559-564.

4. Yesildag A, Yariktas M, Doner F, Aydin G, Munduz M, Topal U (2010) Osteochondroma of the coronoid process and joint formation with zygomatic arch (jacob disease): report of a case. Eur J Dent 4, 91-94.

5. James RB, Alexander RW, Traver JG Jr (1974) Osteochondroma of the mandibular coronoid process. Report of a case. Oral Surg Oral Med Oral Pathol 37, 189-195.

6. Emekli U, Aslan A, Onel D, Cizmeci O, Demiryont M (2002) Osteochondroma of the coronoid process (Jacob's disease). J Oral Maxillofac Surg 60, 1354-1356.

7. Etöz OA, Alkan A, Yikilmaz A (2009) Osteochondroma of the mandibular coronoid process: a rare cause of limited mouth opening. Br J Oral Maxillofac Surg 47, 409-411.

8. Tavassol F, Spalthoff S, Essig H, Bredt M, Gellrich NC, Kokemüller H (2012) Elongated coronoid process: CT-based quantitative analysis of the coronoid process and review of literature. Int J Oral Maxillofac Surg 41, 331-338. 